\title{
A study of the value of the central and peripheral isoptres in assessing visual field progression in the presence of paracentral scotoma measurements
}

\author{
MICHAEL SCHULZER,' FREDERICK S MIKELBERG,' \\ AND STEPHEN M DRANCE' \\ From the 'Departments of Medicine and Statistics, and the 'Department of Ophthalmology, University of \\ British Columbia, Vancouver, BC, Canada
}

\begin{abstract}
SUMmaRY The visual field records of 45 eyes of 45 patients were evaluated to determine whether examination of the peripheral field of vision in patients with glaucoma and field defects adds useful information about the progression of visual field damage. The central scotoma mass, foveal sensitivity, and the central and peripheral mass of the visual field were quantified from measurements on the Tübinger perimeter. Most of the information on visual field progression was contained in the scotoma mass. When both scotoma mass and foveal sensitivity were known, central or peripheral isoptres did not add statistically significant information on progression. Evaluation of scotomas and assessment of central isoptres or of foveal sensitivity made perimetry of the peripheral isoptres redundant. The appearance of fresh peripheral scotomas or the quantification of changes in peripheral scotomas was not examined in the current study, and they would of course be important when they occurred.
\end{abstract}

The examination of the field of vision is important when monitoring patients with glaucoma. Decisions on the adequacy of therapy are often based on whether visual field damage is progressing. The evaluation of the field of vision will always involve some compromise, because the patient's cooperation in visual field testing and the time for perimetry are not unlimited. It is not practicable to examine every possible retinal location in detail. Strategies which will maximise the diagnostic information in a minimum of time have to be developed.

We have previously shown that progressive visual field damage results in an increasing density of existing paracentral scotomas, their enlargement, or the development of new paracentral scotomas in previously unaffected areas of the visual field.' Of those patients showing progressive visual field damage $24 \%$ showed an increase in density of the scotomas as the only manifestation of change. $7 \%$ developed scotomas of larger area, and 14\% developed new scotomas as their only sign of progression. This suggests that, when perimetry is

Correspondence to Dr S M Drance, Eyc Care Centre. 2550 Willow Strect. Vancouver. Canada V5Z,3N9. performed in glaucoma patients, threshold information of disturbed points in a scotoma as well as careful delineation of the area of scotomas and examination of the unaffected areas of the visual field are necessary, because any of these can take place as an isolated sign of progression. The rate of progression of field loss varies between patients. However, most patients whose defects deteriorate show either a linear or a curvilinear mode of visual field progression. ' It is not clear whether examination of the peripheral isoptres in patients with glaucoma and field defects adds useful information about the progression of visual field damage, when careful perimetry of the central isoptre and the quantitative perimetry of all scotomas in the central field are available. Detailed examination of the peripheral isoptres involves further time which could be saved if it is not informative. A retrospective study to try partly to evaluate this question was therefore performed.

\section{Material and methods}

The charts of glaucoma patients for whom demographic data were stored on computerised records 
were reviewed. These computerised records were chosen because of the easy access to data such as visual acuity, intraocular pressure, drugs, and details of any surgery. Only patients with routine chronic open-angle glaucoma and pressures greater than 21 $\mathrm{mmHg}$ were selected. Patients with low tension glaucoma were excluded. All patients were part of one of our practices (SMD) and were followed up routinely. Very few, if any, were followed up because of any particular difficulties with their management. To qualify for the study the patients had to have paracentral or arcuate defects within the central $30^{\circ}$ and 22 months or more of follow-up after the development of the field defects. Forty five eyes of 45 patients (26 males and 19 females) were reviewed. Their ages ranged from 35 to 84 years (mean 61.0 (SD 9.5) years; median age 61.7 years). The follow-up periods ranged from 22 to 182 months (mean 91.2 months (SD 46.3), median 96.() months.)

The fields were recorded both kinetically and statically on the Tübinger perimeter, whose records are large enough for planimetry. All perimetry was performed according to a standard protocol followed in our laboratory. Without a corrective lens the peripheral isoptres were initially plotted with a 10minute target size and at least the 1000 and 100 apostilb intensities. A lens was then inserted into the lens holder and static threshold testing was performed to obtain superior profiles at $45^{\circ}$ and $135^{\circ}$ and inferior profiles at $225^{\circ}$ and $315^{\circ}$. These profiles were obtained with $1^{\circ}$ stimulus separation. Central kinetic perimetry was then performed with a range of stimulus intensities of $3 \cdot 2,6 \cdot 3,10,16,32,100$, and 1000 apostilbs, all with a 10-minute target size. As many stimulus intensities were used as was necessary to quantify completely the size and depth of any existing scotomas and to give a maximum rendering of the isoptres centrally. Static screening was then performed on 75 points at the $2 \frac{1}{2^{\circ}}, 5^{\circ}, 10^{\circ}$, and $15^{\circ}$ circular positions. This was to cover any areas unchecked by static profiles.

All patients had to have an initial visual acuity of $20 / 30$ or better and a final visual acuity of $20 / 40$ or better. The planimetry of the visual field records was carried out on the Zeiss video plan to determine the area of the scotomas within the central $30^{\circ}$. The areas within the central and peripheral isoptres were also examined by planimetry, and the foveal sensitivity was recorded.

Each scotoma was delineated by plotting its size to many stimuli. For a sequence of successively increasing stimuli, scotoma areas $\left(\mathrm{mm}^{2}\right)$ specific to a given stimulus were multiplied by the logarithm of the corresponding stimulus intensity as measured in apostilbs. For example, suppose that the outer rim of a scotoma was outlined by a stimulus of log intensity

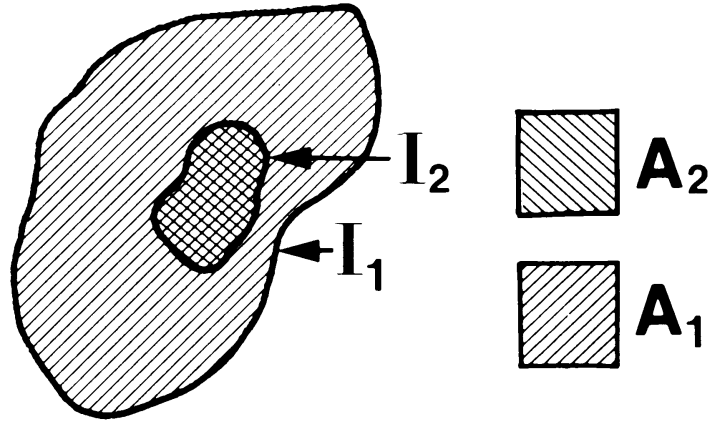

Fig. 1 A schematic representation of a scotoma and the calculation of its mass. A stimulus of log intensity I, has delineated the scotoma shown, enclosing a planimetrically determined total area of $A, \mathrm{~mm}^{2}$. A stronger stimulus, of $\log$ intensity $I_{2}$, has outlined within the first scotoma a deeper region of area $A_{2} \mathrm{~mm}^{2}$. The total scotoma mass was therefore estimated as

$$
\left(A_{1}-A_{2}\right) I_{1}+A_{2} I_{2} m^{2} \times \text { decibels }
$$

$I_{1}$, (Fig. 1), enclosing a total area of $A_{1} \mathrm{~mm}^{2}$, and that the next stronger stimulus, of $\log$ intensity $I_{2},\left(I_{2}>I_{1}\right)$, delineated an inner, deeper scotoma within the first, with an enclosed area of $A_{2} \mathrm{~mm}^{2}\left(A_{2}<A_{1}\right)$. Then the total 'mass' of this scotoma consisted of two components: an outer ring of area $A_{1}-A_{2} \mathrm{~mm}^{2}$ and of 'depth' $I_{1}$, and within it an additional core of area $A_{2} \mathrm{~mm}^{2}$ and of 'depth' $I_{2}$. The masses corresponding to these two components were then summed to yield, for this scotoma, an estimated total mass of

$$
\left(A_{1}-A_{2}\right) I_{1}+A_{2} I_{2}=A_{1} I_{1}+A_{2} I_{2}-A_{2} I_{1}
$$

in $\mathrm{mm}^{2} \times$ decibel units. The sum of all total scotoma masses in the inner $30^{\circ}$ was used to represent the total visual loss for a given field.: Only true scotomas completely surrounded by areas of greater sensitivity were measured. If a scotoma broke through to the periphery it continued to contribute to the total scotoma mass at the level at which it was observed just prior to breakthrough. The position of the isoptres was not used to determine the scotoma mass, and therefore nasal steps or generalised or localised depressions did not enter into the calculation of scotoma mass but would of course influence the measurements made from the relevant isoptres.

All the area measurements used were those plotted on the visual field records of the Tübinger perimeter. These visual field records are flat representations of the spherical retinal surface and are therefore subject to systematic distortions. There is no distortion at the point of fixation which corresponds to the fovea. However, it increases progressively towards the periphery of the visual field in that there is an overestimation of area of loss in the periphery as compared with the corresponding retinal area. Although we were using planimetric measurements 
recorded from both the central and peripheral isoptres, which were therefore subject to these distortions, we disregarded the distortion, since our calculations were not meant to measure the absolute amounts of visual field loss but to evaluate the change of the visual field and the usefulness of information obtained from these records for the follow-up of glaucoma patients. Furthermore, since projection distortions are constant from eye to eye, they were unlikely to affect the intrasubject correlation studies. The visual field records used in Oculus perimetry are equidistant azimuthal polar projections. This projection is equidistant on the meridians only. Areas enclosed by a closed line on the sphere are not directly proportional to the corresponding area on the surface of the projection. With an equivalent azimuthal polar projection the area enclosed by a closed curve on the sphere is directly proportional to the corresponding area on the plane chart. ${ }^{3}$ To obtain a rough approximation of the amount of error introduced into our planimetry calculations with the Oculus perimeter we performed a comparison between the areas enclosed within the central $10^{\circ}$ as compared with the areas enclosed between the 10 and 20 and 20 and $30^{\circ}$. The error obtained was less than $3 \%$.

\section{QUANTIFICATION METHODS}

Five field related parameters in each eye were defined with regard to glaucomatous progression over time.

The foveal sensitivity ('sen') was obtained directly from the static profiles at each field test, and expressed in decibel units (decimal logarithm of apostilbs).

The scotoma mass $(\mathrm{SM})$ in $\mathrm{mm}^{2} \times$ decibel units was calculated from each visual field examination according to the method already fully described. ${ }^{2}$

The central mass $(\mathrm{CM})$ of the island of vision, peripheral mass (PM), and total mass (TM) were defined and quantified. They were determined from each set of perimetric charts, measuring respectively the central (inner $30^{\circ}$ ), peripheral (beyond $30^{\circ}$ ), and total mass of the remaining 'island' of vision. Since the intensity to plot the largest isoptre used was 1000 apostilbs, 'sea level' for the island was set at $\log _{10}$ $1000=3$. This level was recalibrated as 'height 0 ' (Fig. 2 ). The height of the central top of the island was then set to correspond to the logarithm to base 10 of the sensitivity at fixation $\left(0^{\circ}\right)$. Successive concentric isoptre areas corresponding to increasing stimulus intensities were measured planimetrically in $\mathrm{mm}^{2}$ from the $30^{\circ}$ perimetric chart. This information was expressed geometrically, leading to the progressive construction of the central $30^{\circ}$ portion of the island of vision. The top component of island mass was approximated by a cone, its base positioned at a height corresponding to the decimal logarithm of the stimulus intensity of the most central isoptre, and having a circular area corresponding to the planimetrically established area within that isoptre. Its vertex was the point corresponding to the logarithm of sensitivity at fixation. The cone volume (mass) $\left(M_{1}\right.$ in Fig. 2) was then geometrically calculated.

For the purpose of calculating the island, the radius $r$ of the base of this cone had to be measured in degrees from fixation. This was done by calculating planimetrically the total $30^{\circ}$ circular area of the standard $30^{\circ}$ perimetric chart $\left(=25544 \mathrm{~mm}^{2}\right)$, and then solving the proportionate formula,

$$
\frac{\mathrm{r}^{2}}{30^{2}}=\frac{\mathrm{A}_{1}}{25544}
$$

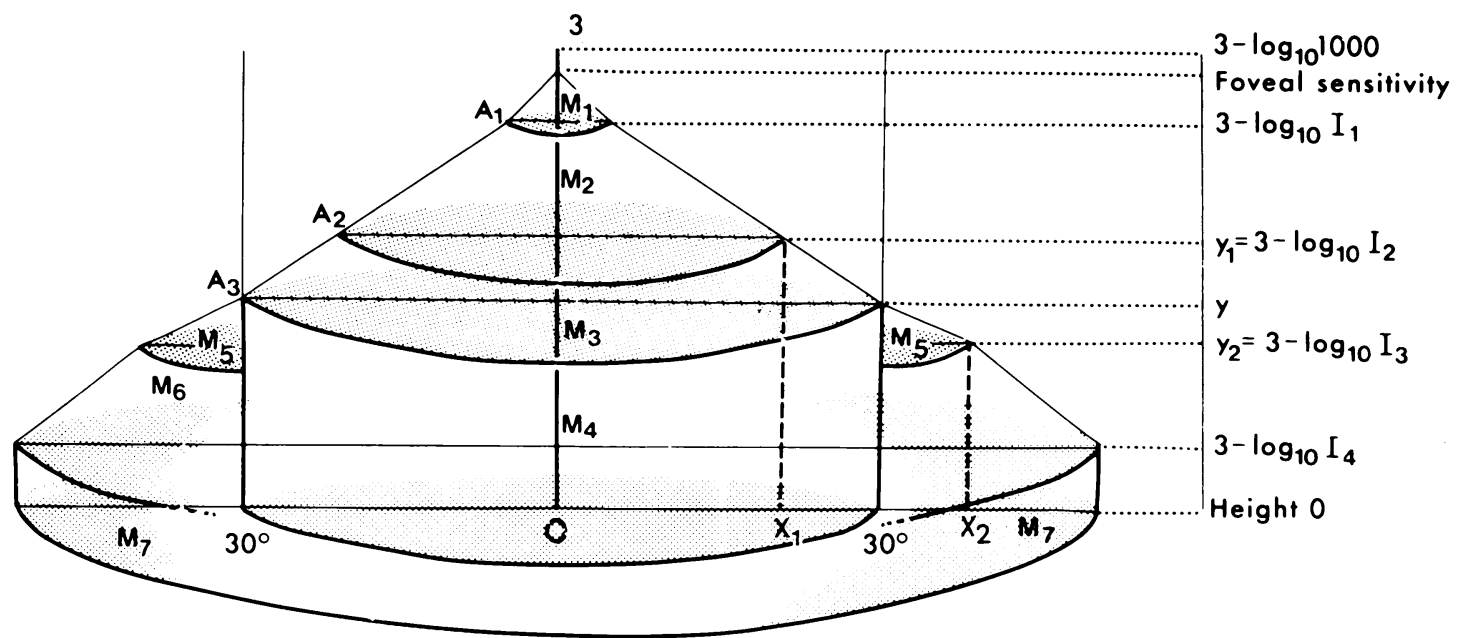

Fig. 2 The quantification of mass of the visual field. 
where $A_{1}$ was the central isoptre area. This was followed by successively increasing isoptre areas to stronger stimuli producing a descending sequence of slices in the shape of truncated cones. The masses of these slices ( $\mathbf{M}_{2}$ in Fig. 1$)$ were calculated geometrically.

The largest central isoptre available was generally well within the $30^{\circ}$ central perimetric circle. To estimate the logarithm of the sensitivity as it would be at $30^{\circ}$ the largest central and the smallest peripheral isoptre heights were used. A scale adjustment had to be introduced, since on the peripheral perimetric chart, constructed on a larger scale, the area of the central $30^{\circ}$ circle of the field was only $2783 \mathrm{~mm}^{2}$. Thus the radius $\mathrm{x}_{1}$ of the largest central isoptre was again expressed in degrees from fixation by solving the formula

$$
\frac{\mathrm{x}_{1}{ }^{2}}{30^{2}}=\frac{\mathrm{A}_{\mathrm{k}}}{25544}
$$

where $A_{k}$ was the area of the largest (k'th) central isoptre. Analogously, the radius $x_{2}$ of the smallest peripheral isoptre, in commensurate units of degrees from fixation, was calculated from

$$
\frac{\mathrm{x}_{2}{ }^{2}}{30^{2}}=\frac{\mathrm{A}_{\mathrm{k}+1}}{2783}
$$

where $A_{k+1}$ was the area of the smallest peripheral isoptre determined from the peripheral chart.

If $y_{1}$ represented the height corresponding to $x_{1}$, and $y_{2}$ corresponding to $x_{2}$, the sensitivity level $y$ at $30^{\circ}$ was then calculated by simple linear interpolation between $y_{1}$ and $y_{2}$. This provided another truncated cone, below which was a cylindrical slice filling out the foundation of the central island out to $30^{\circ}$ (Fig. 2). The component masses obtained from the top cone, the truncated cones, and the bottom cylinder were added to yield an estimated central mass, in $\mathrm{mm}^{2} \times$ decibel units.

A similar technique with some modifications was used to calculate the peripheral mass of the island from which the central mass was removed. The periphery terminated with a centrally hollow truncated cone resting at sea level whenever the largest stimulus used was 1000 apostilbs; otherwise a centrally hollow cylinder formed the foundation of the peripheral island, with a height related to the logarithm of the largest stimulus intensity plotted.

To obtain total island mass the central and peripheral masses were first made comparable by means of a factor which adjusted for the scale difference between the central and the peripheral perimetric charts; they were then added to each other.

It may be noted that the calculation of the total mass of the island was based on the assumption that successive cross-sectional isoptre areas were circular in shape. Although the observed shapes were not always strictly circular, a classical theorem in solid geometry, known as the Cavalieri principle, ${ }^{4}$ guarantees that the circularity assumption yields the correct mass.

\section{STATISTICAL METHODS}

The data to be analysed consisted of 45 eyes of 45 patients, in each of whom a sequence of observations over time was available from which the foveal sensitivity (decimal logarithm), scotoma mass, central mass, peripheral mass, and total mass could be calculated. In addition the initial age and other demographic variables were available.

To examine the progression of any of these variables over time, linear regression on time within patients was carried out, and a weighted time slope was calculated and tested for significance by standard analysis of covariance techniques. ${ }^{5}$ Similar methods were used for correlating any two out of the five variables. Such methods were generally warranted by the reasonably symmetrical, light-tailed distributions of the residuals examined. ${ }^{6}$ To compare how much of the information about progression was contained in each of the five variables, adjustments were first carried out within patients, whereby a set of the variables (or a single variable) were first 'partialled out' by multiple (or simple) regression followed by the calculation of the adjusted residuals. These were then regressed against time within patients, following the same method of analysis of covariance.

\section{Results}

It was clear that progression was demonstrated by SM increasing significantly with time (Table 1$)$. It could also be noted that sen, CM, PM, and TM significantly decreased with time (Tables 2-5).

SM, as expected, was highly negatively correlated with all four of the other field variables (Tables 1, 2). It was further noted that, when adjusted for sen, SM still progressed over time with high statistical significance (Table 1). Similarly, adjusting SM for any of the field mass variables (CM, PM, or TM) reduced the time correlation only slightly (from 0.96 to $0 \cdot 76$ ).

It should also be noted that only the sen time slopes were correlated with age $(p=0.032 ; r=-0.31)$. The mean sen level observed for each patient also correlated highly with age $(p=0.0019 ; r=-0.44)$. None of the other variables was age related. No significant differences in the progression rates for any of the field variables discussed could be found to correspond to the linear or quadratic modes of progression of scotoma masses previously described. ${ }^{2}$ A highly significant positive correlation could be found between all pairs sen, CM, PM, and TM (Tables 2-5). 
Table 1* Scotoma mass (central field) in progression

\begin{tabular}{|c|c|c|c|c|c|c|}
\hline $\begin{array}{l}\text { Dependent } \\
\text { lariable }\end{array}$ & Adjusted for & $\begin{array}{l}\text { Independent } \\
\text { variable }\end{array}$ & Slopet & Correlation ${ }^{\dagger}$ & $\begin{array}{l}\text { Coefficient of } \\
\text { determination }\end{array}$ & $p \ddagger$ \\
\hline \multirow[t]{10}{*}{ SM } & - & $t$ & 24.469 & 0.963 & 0.927 & 0 \\
\hline & $\operatorname{sen}$ & $t$ & $19 \cdot 652$ & 0.728 & 0.530 & 0 \\
\hline & $\mathrm{CM}$ & $t$ & $18 \cdot 065$ & $(0.764$ & 0.584 & 0 \\
\hline & PM & $t$ & $18 \cdot 223$ & 0.758 & 0.574 & 0 \\
\hline & $\mathrm{TM}$ & $t$ & $17 \cdot 374$ & 0.759 & 0.576 & 0 \\
\hline & - & $\mathrm{CM}$ & $-0 \cdot 0859$ & -0.930 & 0.865 & 0 \\
\hline & - & PM & -0.278 & -0.932 & 0.869 & 0 \\
\hline & - & TM & . -0.0259 & -0.933 & 0.870 & 0 \\
\hline & $\mathrm{CM}$ & PM & $-0 \cdot 0460$ & -0.481 & 0.231 & NS \\
\hline & PM & $\mathrm{CM}$ & $0 \cdot 0119$ & 0.597 & 0.356 & NS \\
\hline
\end{tabular}

${ }^{*}$ Adjusted for patient effects.

†Partial slope (correlation) whenever variable has been adjusted in column 2 .

$\ddagger(0)=0 \cdot(00000)$; NS: not significant $(\mathrm{p}>0) \cdot(05)$.

$\mathrm{SM}=$ scotoma mass $\left(\mathrm{mm}^{2} \times\right.$ decibels $) ; \mathrm{t}=$ time (months); $\mathrm{sen}=$ foveal sensitivity $\left(\log _{10}\right) ; \mathrm{CM}=$ central mass $\left(\mathrm{mm}^{2} \times\right.$ decibels $) ; \mathrm{PM}=$ peripheral mass $\left(\mathrm{mm}^{2} \times\right.$ decibels $) ; \mathrm{TM}=$ total mass $\left(\mathrm{mm}^{2} \times\right.$ decibels $)$.

Table ${ }^{*} \quad$ Foveal sensitivity in progression

\begin{tabular}{|c|c|c|c|c|c|}
\hline $\begin{array}{l}\text { Dependent } \\
\text { variable }\end{array}$ & $\begin{array}{l}\text { Independent } \\
\text { variable }\end{array}$ & Slope & Correlation & $\begin{array}{l}\text { Coefficient of } \\
\text { determination }\end{array}$ & $p^{\dagger}$ \\
\hline \multirow[t]{5}{*}{$\operatorname{sen}$} & $t$ & $-0 \cdot 0172$ & $-(0.823$ & 0.677 & 0 \\
\hline & SM & $-0 \cdot(00025$ & -0.698 & 0.487 & $0 \cdot 0001$ \\
\hline & $\mathrm{CM}$ & $0 \cdot 00016$ & $0 \cdot 852$ & 0.726 & 0 \\
\hline & PM & 0.00038 & 0.827 & 0.684 & 0 \\
\hline & $\mathrm{TM}$ & 0.00039 & 0.836 & 0.699 & 0 \\
\hline
\end{tabular}

${ }^{*}$ Adjusted for patient effects.

$+(0)=0 \cdot(00)(0)$.

Abbreviations as in Table 1 .

Table 3* Central field mass in progression

\begin{tabular}{|c|c|c|c|c|c|c|}
\hline $\begin{array}{l}\text { Dependent } \\
\text { variable }\end{array}$ & Adjusted for & $\begin{array}{l}\text { Independent } \\
\text { variable }\end{array}$ & Slopet & Correlationt & $\begin{array}{l}\text { Coefficient of } \\
\text { determination }\end{array}$ & $p \ddagger$ \\
\hline \multirow[t]{7}{*}{$\mathrm{CM}$} & - & $t$ & $-68 \cdot 110$ & -0.833 & 0.694 & 0 \\
\hline & $\operatorname{sen}$ & $t$ & -34.943 & $-0 \cdot 450$ & $0 \cdot 202$ & 0 \\
\hline & SM & $t$ & -15.965 & $-0 \cdot 306$ & 0.094 & 0.016 \\
\hline & $\operatorname{sen}, \mathrm{SM}$ & $t$ & $-8 \cdot 805$ & $-0 \cdot 229$ & $0 \cdot 052$ & NS \\
\hline & SM, PM & $t$ & $2 \cdot 816$ & $0 \cdot 199$ & $0 \cdot 040$ & NS \\
\hline & - & PM & 1.965 & 0.925 & $0 \cdot 856$ & 0 \\
\hline & SM & PM & $1 \cdot 337$ & 0.699 & 0.489 & 0 \\
\hline
\end{tabular}

*Adjusted for patient effects.

†Partial slope (correlation) whenever variable has been adjusted in column 2.

$\ddagger()=0 \cdot(0)(0) 0)$; $N S=$ not significant $(p>0 \cdot(0))$.

Abbreviations as in Table 1.

Table 4* Peripheral field mass in progression

\begin{tabular}{|c|c|c|c|c|c|c|}
\hline $\begin{array}{l}\text { Dependent } \\
\text { variable }\end{array}$ & Adjusted for & $\begin{array}{l}\text { Independent } \\
\text { variable }\end{array}$ & Slopet & Correlationt & $\begin{array}{l}\text { Coefficient of } \\
\text { determination }\end{array}$ & $p \ddagger$ \\
\hline \multirow[t]{6}{*}{ PM } & - & $t$ & $-20 \cdot 897$ & -0.782 & $0 \cdot 612$ & 0 \\
\hline & $\operatorname{sen}$ & $t$ & $-13 \cdot 712$ & -0.446 & $0 \cdot 199$ & 0 \\
\hline & SM & $t$ & $-4 \cdot 685$ & -0.343 & $0 \cdot 118$ & 0.04 \\
\hline & sen, SM & $t$ & $-3 \cdot 742$ & $-0 \cdot 274$ & 0.075 & NS \\
\hline & SM. CM & $t$ & -1.726 & $-0 \cdot 277$ & 0.077 & NS \\
\hline & SM & $\mathrm{CM}$ & $0 \cdot 144$ & $0 \cdot 730$ & 0.533 & 0 \\
\hline
\end{tabular}

${ }^{*}$ Adjusted for patient effects.

†Partial slope (correlation) whenever variable has been adjusted in column 2.

$\ddagger()=() \cdot()(0)(0) ; \mathrm{NS}=$ not significant $(p>0) \cdot(0) 5)$.

Abbreviations as in Table 1. 
Table $5^{*}$ Total field mass in progression

\begin{tabular}{|c|c|c|c|c|c|c|}
\hline $\begin{array}{l}\text { Dependent } \\
\text { variable }\end{array}$ & Adjusted for & $\begin{array}{l}\text { Independent } \\
\text { variable }\end{array}$ & Slopet & Correlationt & $\begin{array}{l}\text { Coefficient of } \\
\text { determination }\end{array}$ & $p \ddagger$ \\
\hline \multirow[t]{4}{*}{$\mathrm{TM}$} & - & $t$ & -259.91 & -0.814 & 0.662 & 0 \\
\hline & $\operatorname{sen}$ & $t$ & $-160 \cdot 80$ & -0.462 & 0.213 & 0 \\
\hline & SM & $t$ & -58.96 & -0.342 & 0.117 & 0.02 \\
\hline & sen, SM & $t$ & $-43 \cdot 16$ & -0.265 & 0.070 & NS \\
\hline
\end{tabular}

*Adjusted for patient effects.

†Partial slope (correlation) whenever variable has been adjusted in column 2 .

$\ddagger(0)=(0)(0) 00 ; \mathrm{NS}=$ not significant $(\mathrm{p}>0) \cdot(0) 5)$.

Abbreviations as in Table 1 .

\section{Discussion}

In spite of the fact that patients were selected into this study who maintained a fairly constant visual acuity throughout the follow-up period, a marked, highly statistically significant progression can be observed over time in all visual field parameters (but not all patients). It is apparent, however, that among the parameters considered the paracentral scotoma mass contains most of the information about progression and is hardly affected by the additional knowledge of the central or peripheral isoptres. On the other hand the information bearing on progression contained in any of the other visual field mass measurements considered here is only slightly significant when the paracentral scotoma mass is known and adjusted for, and becomes non-significant when both the scotoma mass and foveal sensitivity are recorded.

It is also interesting to note that the peripheral mass sheds no further light concerning progression when the paracentral scotoma mass and the central mass are known. It should also be noted that the central mass gives no further information with respect to progression when both the paracentral scotoma mass and the peripheral mass of the visual field are known. This, however, would not be the case in standard perimetry, which concentrates almost exclusively on the inner $30^{\circ}$ of the field.

While the paracentral scotoma mass carries the major information regarding the progression of the visual field in glaucoma, any one of the other measures-foveal sensitivity, central mass, or peripheral mass-carries some additional informa- tion about progression of field loss. It is therefore likely that they are all manifestations of a diffuse change which affects them all.

It would appear that in perimetry of glaucomatous progression the accurate quantitation of paracentral scotomas and the assessment of the central isoptres or foveal sensitivity make perimetry of the peripheral isoptres redundant. It must be stressed that the present study did not consider the appearance of fresh peripheral scotomas, which would be important when they occurred. The examination of the peripheral field for the occurrence of fresh scotomas or change in existing scotomas in the peripheral field should still not be abandoned until specific investigation of the peripheral scotomas has been undertaken.

This study was supported by the Medical Research Council of Canada, grant number MT 1578.

\section{References}

1 Mikelberg FS, Drance SM. The mode of progression of visual field defects in glaucoma. Am J Ophthalmol 1984: 98: 443-5.

2 Mikelberg FS, Schulzer M. Drance SM. Lau W. The rate of progression of scotomas in glaucoma. A $m$ J Ophthalmol 1986: 101: 1-6.

3 Doesschate JT. Perimetric charts in equivalent projection allowing a planimetric determination of the extension of the visual field. Ophthalmologica 1947; 113: 257-70).

4 Eves HW. An introduction to the history of mathematics. 5th ed. Philadelphia: Saunders College Publication, 1983: 294-7.

5 Draper NR. Smith H. Applied regression analysis. 2nd ed. New York: Wiley, 1981: 241-57.

6 Draper NR. Smith H. Applied regression analysis. 2nd ed. New York: Wiley, 1981: 141-92.

Accepted for publication 1.3 August 1986. 